\title{
A FINITE ELEMENT ANALYSIS OF BEAMS ON ELASTIC FOUNDATION INCLUDING SHEAR AND AXIAL EFFECTS
}

\author{
Zissimos P. Mourelatos $\dagger$ and Michael G. Parsons $\ddagger$ \\ Department of Naval Architecture and Marine Engineering, The University of Michigan, Ann Arbor, \\ MI 48109, U.S.A.
}

(Received 24 May 1985)

\begin{abstract}
A displacement finite element method for analyzing a beam on continuous elastic foundation is presented. A three-dimensional model which accounts for the effects of both the Filonenko-Borodich and Pasternak foundation models in a consistent and complete way is used. A variational principle is introduced with the slope field due to bending only and the displacement field approximated by independent quantities subjected to variation. Numerical examples illustrate the accuracy of the element, the importance of shear, axjal and shear-axial interaction effects associated with continuous elastic foundation, and finally the application of the element to a rotor supported by two hydrodynamic journal bearings.
\end{abstract}

\section{NOTATION}

$b_{y}, b_{z}$

D

$E$

$f_{y}, f_{z}$

$f_{y z}, f_{z y}$

G

$I_{y}, I_{z}$

$I_{y z}$

$k$

$L^{2}$

$M_{2}$

$N_{x}^{2}$
$N_{x}^{c r}$

$N_{x}^{c r}$

$p_{y}, p$

$Q_{y}, Q$

$u$

$u_{0}$

$\bar{v}$

$V_{y}, V_{z}$

$w$

$\bar{w}$

$x, y, z$

$\gamma_{x y}$

$\delta_{y}, \delta_{z}$

$\theta_{z} \quad$ angle of rotation of the beam about the $y$ axis $\bar{\theta}_{2}$

beam cross sectional area

definition in Fig. 1

beam diameter

Young's modulus of elasticity

uniform, elastic foundation spring constants

per unit length in $y$ and $z$ directions

uniform, coupling elastic foundation spring constants per unit length in $y$ and $z$ directions shear modulus

moments of inertia of the beam cross section with respect to $y$ and $z$ axes

cross moment of inertia of the beam cross section

shear correction factor with respect to $z$ axis beam element length

bending moment in $x-y$ plane

axial force; positive in tension

critical axial force (buckling load)

$i=1,2,3$ shape functions

uniform distributed loads in $y$ and $z$ directions

moments of area with respect to $y$ and $z$ axes

(Fig. 1)

axial displacement of the beam

axial displacement of the centroidal axis of the beam

displacement of the beam in $y$ direction

virtual displacement of the beam in $y$ direction

shear forces in $y$ and $z$ directions

dispiacement of the beam in $z$ direction

virtual displacement of the beam in $z$ direction coordinate system

shear strain in $y$ direction on a cross section perpendicular to $x$ direction

translations of the elastic foundation in the $y$ and $z$ directions due to bending only

virtual angle of rotation of the beam about the $y$ axis due to bending only

† Present address: General Motors Research Laboratories, Fluid Mechanies Department, Warren, MI 48090; formerly Ph.D. Candidate.

$\ddagger$ Professor and Chairman.

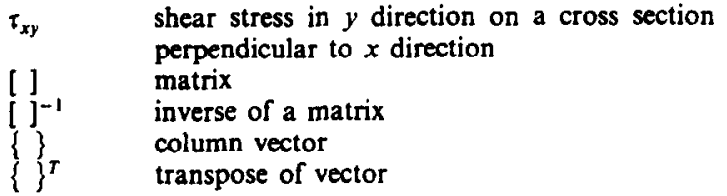

\section{INTRODUCTION}

The problem of a beam (or plate) on an elastic foundation is important in both the civil and mechanical engineering fields, since it constitutes a practical idealization for many problems (e.g. the behavior of a shaft within a hydrodynamically lubricated bearing, a floating body on the water, etc.). The importance of the problem is indicated by the large number of papers which have appeared in the literature about beams on elastic foundation. Some of these papers are very recent [1-5].

Generally, the analysis of bending of beams on an elastic foundation is developed on the assumption that the reaction forces of the foundation are proportional at every point to the deflection of the beam at that point. This simple representation of elastic foundation was introduced by Winkler [6], who assumed that the base consisted of closely spaced, independent

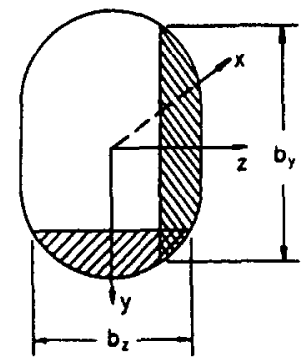

Fig. 1. Definition of $b_{z}$ and $b_{y}$. 

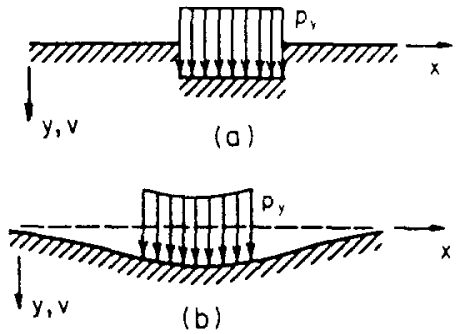

Fig. 2. Deflections of elastic foundations under uniform pressure $p_{y}$. (a) Winkler Foundation. (b) Elastic solid [6].

linear springs as in Fig. 2(a). As a next step, the foundation was considered as an elastic continuum taking the displacement pattern of Fig. 2(b) under the applied load $p$. However, the Winkler foundation could not predict accurately the displacement of some solids, e.g. soil [6]. In an attempt to find a physically close and mathematically simple representation for these materials, other types of foundation have been introduced. The Filonenko-Borodich foundation [6] introduces a stretched elastic membrane subjected to a constant-tension field $N_{x}$ at the top ends of the springs, in order to achieve some degree of interaction between the spring elements as in Fig. 3. The Pasternak foundation $[6-10]$ assumes the existence of shear interaction between the spring elements. This may be accomplished by connecting the ends of the springs with a beam consisting of incompressible vertical elements which deform only by transverse shear (Fig. 4).

Establishing more realistic foundation models and developing simplified methods for analyzing structures which take into account the elasticity of the foundations are among the modern trends in the theory of structures on elastic foundations [9].

An approximate numerical approach, based on the finite element technique, is appropriate for analyzing structures on an elastic foundation, since it leads to relatively straightforward matrix expressions. For the computations of the long and complicated numerical results, one can rely upon the digital computer. Besides, while the geometry of the structure, the loading and boundary conditions make the mathematical solution of the general problem in closed form almost impossible [7], in the versatile finite element method they can be completely arbitrary $[9,10]$.

In the present paper, a finite element displacement method is presented to analyze the problem of a beam on a general elastic foundation which includes

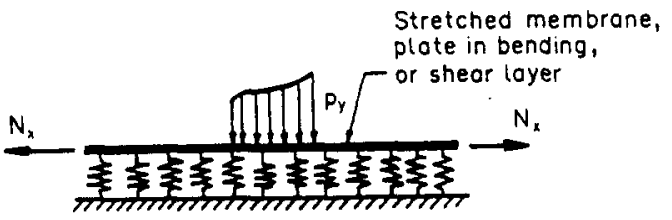

Fig. 3. Filonenko-Borodich elastic foundation [6].

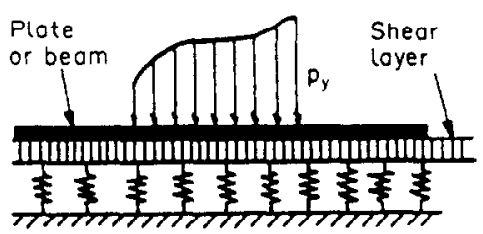

Fig. 4. Pasternak elastic foundation [6].

the effects of both the Filonecko-Borodich and Pasternak foundation models. The problem is formulated as a coupled, three-dimensional problem. A beam with symmetric cross section with respect to both the vertical and horizontal planes is on a general type of continuum elastic foundation. This foundation representation has separate, uniform spring constants per unit length in the vertical and horizontal planes and uniform coupling spring constants between the two planes. The proposed beam element on elastic foundation is important in the area of rotor dynamics, where the behavior of flexible shafts within hydrodynamically lubricated journal bearings is analyzed $[11,12]$. The nonlinear hydrodynamic characteristics of a journal bearing can be represented by elastic foundation with spring constants in two perpendicular planes and coupling spring constants between these two planes. An extension of the present static model to include the dynamic effects of lateral and rotational inertias is presented in [13].

Finite element beam formulations in the literature (e.g. [5, 14-17]) include bending, shear, axial loading and elastic foundation, but typically not simultaneously and without a complete and consistent treatment of the coupling effects among the various loadings.

\section{BASIC ASSUMPTIONS AND DEFINITIONS}

Within the limits of elementary beam theory, it is possible to include the effects of bending, shear and axial force in the stiffness matrix of a beam on elastic foundation. The analysis will be based on the following assumptions:

1. Plane sections remain plane before and after deformation (no warping).

2. Strains are sufficiently small that the cross sectional geometry does not change (no Poisson effects).

3. Beam slopes are small.

4. Symmetric beam cross section with respect to both vertical and horizontal planes.

5. Beam material is linearly elastic, homogeneous, isotropic and continuous.

The definition of the coordinate system adopted for the beam is given in Fig. 5 .

From the geometry of the beam bending in the $x-y$ plane (vertical plane) under the assumptions 1,2 and 

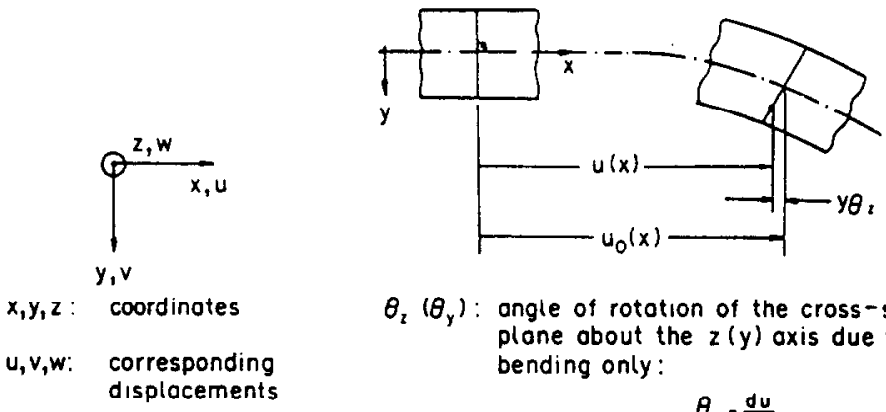

$\theta_{z}\left(\theta_{y}\right)$ : angle of rototion of the cross-sectional plone obout the $z(y)$ axis due to pure bending only:

$$
\theta_{2}=\frac{d u}{d x}
$$

$u_{0}(x)$ : axial displacement of the centroidal axis

Fig. 5. Beam differential element coordinate system.

3 , the axial displacement $u$ due to bending only can be expressed as:

$$
u(x)=-y \theta_{z}(x)-z \theta_{y}(x) .
$$

Including shear and axial effects, the general expression for the distribution of the average shear stress on a cross section is given by $[18,19]$

$$
\begin{aligned}
\tau_{x y}=\left[\frac{Q_{z} I_{y}-Q_{y} I_{y z}}{b_{z}\left(I_{y} I_{z}-I_{y z}^{2}\right)}\right]\left\{V_{y}-N_{x} \frac{\mathrm{d} v}{\mathrm{~d} x}\right\} \\
+\left[\frac{Q_{y} I_{z}-Q_{z} I_{y z}}{b_{z}\left(I_{y} I_{z}-I_{y z}^{2}\right)}\right]\left\{V_{z}-N_{x} \frac{\mathrm{d} w}{\mathrm{~d} x}\right\},
\end{aligned}
$$

which, after the application of the beam cross section symmetry assumption, becomes:

$$
\tau_{x y}=\frac{Q_{z}}{b_{z} I_{z}}\left(V_{y}-N_{x} \frac{\mathrm{d} v}{\mathrm{~d} x}\right)+\frac{Q_{y}}{b_{z} I_{y}}\left(V_{z}-N_{x} \frac{\mathrm{d} w}{\mathrm{~d} x}\right) .
$$

Equivalently, the shear strain is

$$
\begin{aligned}
\tau_{x y}=\frac{\tau_{x y}}{G}=\frac{Q_{z}}{b_{z} I_{z} G}\left(V_{y}\right. & \left.-N_{x} \frac{\mathrm{d} v}{\mathrm{~d} x}\right) \\
& +\frac{Q_{y}}{b_{z} I_{y} G}\left(V_{z}-N_{x} \frac{\mathrm{d} w}{\mathrm{~d} x}\right) .
\end{aligned}
$$

The linear approximation for the shear strain is

$$
\gamma_{x y}=\frac{\mathrm{d} u}{\mathrm{~d} y}+\frac{\mathrm{d} v}{\mathrm{~d} x}
$$

Equations (1), (3) and (4) imply:

$$
\begin{aligned}
\frac{Q_{z}}{b_{z} I_{z} G}\left(V_{y}\right. & \left.-N_{x} \frac{\mathrm{d} v}{\mathrm{~d} x}\right) \\
& +\frac{Q_{y}}{b_{z} I_{y} G}\left(V_{z}-N_{x} \frac{\mathrm{d} w}{\mathrm{~d} x}\right)=-\theta_{z}+\frac{\mathrm{d} v}{\mathrm{~d} x},
\end{aligned}
$$

and since the shaft cross section is symmetric with respect to the $y$ axis,

$$
Q_{y}=0
$$

this expression simplifies to

$$
\frac{Q_{z}}{b_{z} I_{z} G}\left(V_{y}-N_{x} \frac{\mathrm{d} v}{\mathrm{~d} x}\right)=-\theta_{z}+\frac{\mathrm{d} v}{\mathrm{~d} x} .
$$

The $Q_{z} / b_{z}$ term of eqn (5) depends on the coordinate $y$. To simplify this situation, we replace $\tau_{x y}$ by a uniform stress distribution and account for its variation with $y$ by introducing a shear correction factor $k_{z}$. This factor $k_{z}$ is defined in [19] as the constant by which the average shear strain must be multiplied in order to obtain an equivalent uniform shear strain producing the same shear displacement as the distributed shear strain of the left hand side of eqn (5). With this definition,

$$
k_{z}=\frac{A}{I_{z}^{2}} \int_{A}\left(\frac{Q_{2}}{b_{2}}\right)^{2} \mathrm{~d} A
$$

where $A$ is the cross sectional area. Values of $k_{z}$ for typical symmetric cross sections [19] are $6 / 5$ for rectangular sections, $10 / 9$ for circular sections, and near unity for $I$ sections. Using eqn (6), eqn (5) becomes

$$
\theta_{z}=\left(1+\frac{k_{z} N_{x}}{A G}\right) \frac{\mathrm{d} v}{\mathrm{~d} x}-\frac{k_{z} V_{y}}{A G}
$$

Notice that the total rotation angle for the beam elastic curve is $\mathrm{d} v / \mathrm{d} x$ for the $x-y$ plane, while $\theta_{z}$ is the part of $\mathrm{d} v / \mathrm{d} x$ due to bending only. A similar expression is valid for $\theta_{y}$ in the $x-z$ plane (horizontal plane).

\section{BOUNDARY VALUE PROBLEM FOR} THE BEAM ELEMENT

The beam differential element shown in Fig. 6 is used for the $x-y$ plane. The equilibrium of forces with respect to the $y$-axis gives:

$$
\frac{\mathrm{d} V_{y}}{\mathrm{~d} x}=-\left(p_{y}-f_{y} v+f_{y} \delta_{y}-f_{y z} w+f_{y z} \delta_{z}\right)
$$

The moment equilibrium with respect to the left end 


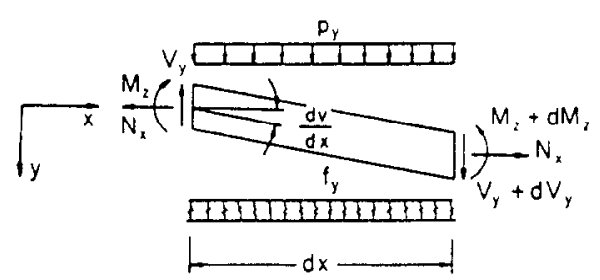

Fig. 6. Uniform differential beam element with uniform distributed load on elastic foundation ( $x-y$ plane).

of the beam element shown in Fig. 6 gives

$$
V_{y}=\frac{\mathrm{d} M_{z}}{\mathrm{~d} x}+N_{x} \frac{\mathrm{d} v}{\mathrm{~d} x} .
$$

Since the beam cross section has been assumed to be symmetric, the constitutive relation for the beam bending based on Hooke's law is

$$
M_{2}(x)=-E I_{z} \frac{\mathrm{d} \theta_{2}(x)}{\mathrm{d} x} .
$$

Differentiation of eqn (9) with respect to $x$ and application of eqn (7) gives the governing differential equation of the beam element for the $x-y$ plane:

$$
\frac{\mathrm{d} V_{y}}{\mathrm{~d} x}=\left(1+\frac{k_{z} N_{x}}{A G}\right) \frac{\mathrm{d}^{2} M_{z}}{\mathrm{~d} x^{2}}+N_{x} \frac{\mathrm{d} \theta_{z}}{\mathrm{~d} x} .
$$

A similar expression is valid for the $x-z$ plane.

Notice from eqns (8) and (11) that if the uniform coupling elastic foundation constant $f_{y z}$ is not zero the problem is coupled between the $x-y$ and $x-z$ planes.

For reasons of convenience, the notation of Fig. 7 will be used for the derivation of the stiffness matrix and load vector of the proposed beam finite element.

\section{DISPLACEMENT FIELD}

It is common to approximate the displacement field along a beam element with a cubic polynomial taking as nodal degrees of freedom the displacement and rotation at the beam ends $[5,14,15,16]$. This approximation is very accurate for free beams, but not so accurate for beams on elastic foundation [1]. However, when the beam is on elastic foundation, the shear force is a function of the displacement [eqn (8)].Therefore, the angle of rotation $\theta_{2}$ of the beam elastic curve due to bending only is dependent on the displacement according to eqn (7). This does not allow the use of the standard degrees of freedom of

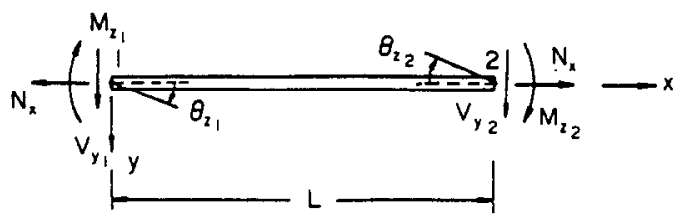

Fig. 7. Notation for the beam finite element in $x-y$ plane. the displacement and rotation at each end of the beam element. Instead, independent approximations are used for the rotation 0 : and the displacement $v$.

A linear approximation for both $v$ and $\theta_{z}(x-y$ plane) and $w$ and $\theta_{y}$ ( $x-z$ plane) did not give a good accuracy in calculating the deformation of a simply supported beam on an elastic foundation $\left(f_{y} \neq 0\right.$, $f_{z}=f_{y z}=f_{z y}=0$ ) using a reasonable number of elements. The accuracy of the solution was obtained comparing the numerical solution with the analytical solution. For this reason, the displacement $v$ and the virtual displacement $\bar{v}$ along the length of the element are approximated by a quadratic polynomial as:

$$
v(x)=\left\{N_{1}(x) N_{2}(x) N_{3}(x)\right\}\left[\begin{array}{l}
v_{1} \\
v_{2} \\
v_{3}
\end{array}\right]
$$

where $v_{1}, v_{2}$ and $v_{3}$ are the nodal displacements at the two ends and the middle of the element, respectively (Fig. 8), and

$$
\begin{aligned}
N_{1}(x) & =1-3 \zeta+2 \zeta^{2} \\
N_{2}(x) & =-\zeta+2 \zeta^{2} \\
N_{3}(x) & =4 \zeta-4 \zeta^{2} \\
\zeta & =\frac{x}{L}
\end{aligned}
$$

are the shape or interpolation functions. The slope approximation is

$$
\theta_{7}(x)=\left\{N_{1}(x) N_{2}(x) N_{3}(x)\right\}\left[\begin{array}{l}
\theta_{z_{1}} \\
\theta_{z_{2}} \\
\theta_{z_{3}}
\end{array}\right]
$$

Similar expressions can be obtained for the $x-z$ plane.

\section{WEAK FORMULATION AND DISCRETIZED SYSTEM}

The weak formulation is a variational form of the given boundary value problem for each element, transforming the true continuous displacement field into a discrete approximation using the nodal displacements and rotations as given by eqns (12) and (14). The Galerkin method [20] is used to obtain that variational form. Introducing the approximating displacement and rotation field into the governing differential equation, a residual is produced since it does not satisfy the boundary value problem exactly.

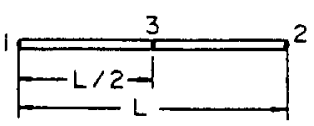

Fig. 8. Nodal points for the beam finite element. 
Galerkin's method makes this residual orthogonal to the approximating (shape) functions over the length of the element. There is no limit on the highest-order derivative which appears in the weak form. For the integrals in the weak form to exist, however, it cannot exceed the order of the continuity of the interpolation functions by more than one [21]. With the interpolation functions of eqns (12) and (14), the continuity of the displacement field $v(x)(w(x))$ and the rotation field $\theta_{z}(x)\left(\theta_{y}(x)\right)$ is ensured. This implies that at most first-order derivatives of the displacement and rotation may appear in the integral formulation obtained by Galerkin's method. For this reason, integration by parts is applied where it is needed.

If we multiply the governing differential eqn (1I) for the $x-y$ plane by the virtual displacement $\bar{v}$, and integrate over the beam length, we obtain:

$$
\begin{aligned}
\int_{0}^{L} \frac{\mathrm{d} V_{y}}{\mathrm{~d} x} \bar{v} \mathrm{~d} x-\int_{0}^{L}\left(1+\frac{k_{z} N_{x}}{A G}\right) \frac{\mathrm{d}^{2} M}{\mathrm{~d} x^{2}} \bar{v} \mathrm{~d} x \\
-\int_{0}^{L} N_{x} \frac{\mathrm{d} \theta_{z}}{\mathrm{~d} x} \bar{v} \mathrm{~d} x=0 .
\end{aligned}
$$

Integration by parts of the second term twice, and of the third term once, and use of eqn (7) to eliminate $\mathrm{d}^{2} \bar{v} / \mathrm{d} x^{2}$ and $\mathrm{d} \bar{v} / \mathrm{d} x$ yield:

$$
\begin{aligned}
& -\int_{0}^{L} \frac{\mathrm{d} V_{y}}{\mathrm{~d} x} \bar{v} \mathrm{~d} x+\int_{0}^{L} M_{z} \frac{\mathrm{d} \bar{\theta}_{z}}{\mathrm{~d} x} \mathrm{~d} x \\
& -\left(1+\frac{k_{z} N_{x}}{A G}\right)^{-1} \int_{0}^{L} N_{x} \theta_{z} \bar{\theta}_{z} \mathrm{~d} x \\
& -\left.M_{z} \bar{\theta}_{z}\right|_{0} ^{L}+\left.V_{y} \bar{v}\right|_{0} ^{L}-\frac{k_{z}}{A G} \\
& \quad \times\left(1+\frac{k_{z} N_{x}}{A G}\right)^{-1} \int_{0}^{L} N_{x} \theta_{z} \bar{V} \mathrm{~d} x \\
& +\frac{k_{z}}{A G} \int_{0}^{L} M_{z} \frac{\mathrm{d} \bar{D}_{y}}{\mathrm{~d} x} \mathrm{~d} x-\left.M_{z} \frac{k_{z} \bar{V}_{y}}{A G}\right|_{0} ^{L}=0 .
\end{aligned}
$$

If we combine the last two terms by integration by parts, the last three terms of the above eqn (15) become equal to:

$$
\begin{aligned}
-\frac{k_{z}}{A G}\left(1+\frac{k_{z} N_{x}}{A G}\right)^{-1} \int_{0}^{L} D_{y} \\
\quad \times\left[N_{x} \theta_{z}+\left(1+\frac{k_{z} N_{x}}{A G}\right) \frac{\mathrm{d} M_{z}}{\mathrm{~d} x}\right] \mathrm{d} x
\end{aligned}
$$

or

$$
-\frac{k_{z}}{A G}\left(1+\frac{k_{z} N_{x}}{A G}\right)^{-1} \int_{0}^{L} V_{y} D_{y} \mathrm{~d} x
$$

since elimination of $d v / d x$ in eqn (9) using eqn (7) gives:

$$
V_{y}=\left(1+\frac{k_{*} N_{x}}{A G}\right) \frac{\mathrm{d} M_{z}}{\mathrm{~d} x}+N_{x} 0_{z}
$$

Therefore, eqn (15) is equal to:

$$
\begin{aligned}
& -\int_{0}^{L} M_{z} \frac{\mathrm{d} \bar{\theta}_{z}}{\mathrm{~d} x} \mathrm{~d} x+\left(1+\frac{k_{z} N_{x}}{A G}\right)^{-1} \\
& \times \int_{0}^{L} N_{x} \theta_{z} \bar{\theta}_{z} \mathrm{~d} x+\left(1+\frac{k_{z} N_{x}}{A G}\right)^{-1} \\
& \times \frac{k_{z}}{A G} \int_{0}^{L} V_{y} \nabla_{y} \mathrm{~d} x \\
& =-\int_{0}^{L} \frac{\mathrm{d} V_{y}}{\mathrm{~d} x} \bar{v} \mathrm{~d} x-\left.M_{z} \bar{\theta}_{z}\right|_{0} ^{L}+\left.V_{y} \bar{v}\right|_{0} ^{L} .
\end{aligned}
$$

By substitution of the shear force $V_{y}$ from eqn (7), the bending moment $M_{z}$ from eqn (10) and the derivative of the shear force $\mathrm{d} V_{y} / \mathrm{d} x$ from eqn (9), and by adoption of the Fig. 7 notation, the weak form of eqn (16) takes the following final form:

$$
\begin{aligned}
E I_{z} \int_{0}^{L} & \frac{\mathrm{d} \theta_{z}}{\mathrm{~d} x} \frac{\mathrm{d} \bar{\theta}_{z}}{\mathrm{~d} x} \mathrm{~d} x+\left(1+\frac{k_{z} N_{x}}{A G}\right)^{-1} \\
& \times\left(N_{x}+\frac{A G}{k_{z}}\right) \int_{0}^{L} \theta_{z} \bar{\theta}_{z} \mathrm{~d} x+\left(1+\frac{k_{z} N_{x}}{A G}\right) \\
& \times \frac{A G}{k_{z}} \int_{0}^{L} \frac{\mathrm{d} v}{\mathrm{~d} x} \frac{\mathrm{d} \bar{v}}{\mathrm{~d} x} \mathrm{~d} x-\frac{A G}{k_{z}} \int_{0}^{L} \frac{\mathrm{d} v}{\mathrm{~d} x} \bar{\theta}_{z} \mathrm{~d} x \\
& -\frac{A G}{k_{z}} \int_{0}^{L} \theta_{z} \frac{\mathrm{d} \bar{v}}{\mathrm{~d} x} \mathrm{~d} x+f_{y} \int_{0}^{L} v \bar{v} \mathrm{~d} x \\
& +f_{y z} \int_{0}^{L} w \bar{v} \mathrm{~d} x=\int_{0}^{L}\left(p_{y}+f_{y} \delta_{y}+f_{y z} \delta_{z}\right) \bar{v} \mathrm{~d} x \\
& +V_{y_{1}} \bar{v}_{1}+V_{y_{2}} \bar{D}_{2}+M_{z_{1}} \theta_{z 1}+M_{z 2} \bar{\theta}_{z 2} .
\end{aligned}
$$

A similar expression holds for the $x-z$ plane.

Using the approximating displacement and rotation fields of eqns (12) and (14) and performing the integrations in the weak forms of both $x-y$ and $x-z$ planes, the element equations for both planes are as follows:

$$
\begin{gathered}
{\left[\begin{array}{cccc}
{\left[\mathrm{SE}_{11}\right]} & {\left[\mathrm{SE}_{12}\right]} & 0 & 0 \\
{\left[\mathrm{SE}_{12}\right]^{T}} & {\left[\mathrm{SE}_{22}\right]} & 0 & {\left[\mathrm{SE}_{24}\right]} \\
0 & 0 & {\left[\mathrm{SE}_{33}\right]} & {\left[\mathrm{SE}_{34}\right]} \\
0 & {\left[\mathrm{SE}_{42}\right]} & {\left[\mathrm{SE}_{34}\right]^{T}} & {\left[\mathrm{SE}_{44}\right]}
\end{array}\right]} \\
\times\left[\begin{array}{c}
\left\{\theta_{z}\right\} \\
\{v\} \\
\left\{\theta_{y}\right\} \\
\{w\}
\end{array}\right]=\left[\begin{array}{c}
\{0\} \\
\left\{d_{1}\right\} \\
\{0\} \\
\left\{d_{2}\right\}
\end{array}\right]+\left[\begin{array}{l}
\left\{M_{z}\right\} \\
\left\{V_{y}\right\} \\
\left\{M_{y}\right\} \\
\left\{V_{z}\right\}
\end{array}\right]
\end{gathered}
$$


where

$$
\begin{aligned}
& \left\{\theta_{z}\right\}=\left\{\theta_{z 1} \theta_{z_{2}} \theta_{z 3}\right\}^{T} \\
& \{v\}=\left\{v_{1} v_{2} v_{3}\right\}^{T} \\
& \left\{\theta_{y}\right\}=\left\{\theta_{y_{1}} \theta_{y_{2}} \theta_{y_{3}}\right\}^{T} \\
& \left\{w^{\prime}\right\}=\left\{w_{1} w_{2} w_{3}\right\}^{T} \\
& \left\{M_{z}\right\}=\left\{M_{z_{1}} M_{z 2} 0\right\}^{T} \\
& \left\{V_{y}\right\}=\left\{V_{y_{1}} V_{y_{2}} 0\right\}^{T} \\
& \left\{M_{y}\right\}=\left\{M_{y_{1}} M_{y_{2}} 0\right\}^{T} \\
& \left\{V_{z}\right\}=\left\{V_{z_{1}} V_{z_{2}} 0\right\}^{T} .
\end{aligned}
$$

The matrices $\left[S E_{11}\right],\left[S E_{12}\right],\left[S E_{22}\right],\left[S E_{24}\right],\left[S E_{33}\right]$, $\left[S E_{34}\right],\left[S E_{44}\right]$ and $\left[S E_{42}\right]$ and the distributed loading vectors $\left\{d_{1}\right\}$ and $\left\{d_{2}\right\}$ are given in [22]. Rearranging eqn (18) we obtain

$$
\left[\frac{\left[K_{r}\right] \mid\left[K_{r e}\right]}{\left[K_{e r}\right] \mid\left[K_{e e}\right]}\right]\left[\frac{\left\{d_{r}\right\}}{\left\{d_{e}\right\}}\right]=\left[\frac{\left\{r_{r_{1}}\right\}}{\left\{r_{e}\right\}}\right]+\left[\frac{\left\{r_{r_{2}}\right\}}{\{0\}}\right],
$$

where

$$
\begin{aligned}
& \left\{d_{r}\right\}=\left\{v_{1} \theta_{z_{1}} w_{1} \theta_{y_{1}} v_{2} \theta_{z_{2}} w_{2} \theta_{y_{2}}\right\}^{T} \\
& \left\{d_{e}\right\}=\left\{v_{3} \theta_{z 3} w_{3} \theta_{y_{3}}\right\}^{T} \\
& \left\{r_{r_{2}}\right\}=\left\{V_{y_{1}} M_{z_{1}} V_{z_{1}} M_{y_{1}} V_{y_{2}} M_{z_{2}} V_{z_{2}} M_{y_{2}}\right\}^{T} .
\end{aligned}
$$

The matrices $\left[K_{r r}\right],\left[K_{r e}\right],\left[K_{e r}\right]$ and $\left[K_{e e}\right]$ and the loading vectors $\left\{r_{r}\right]$ and $\left\{r_{e}\right\}$ are given again in [22].

Elimination of the internal degrees of freedom $\left\{d_{e}\right\}$ from eqn (19) by static condensation gives the final form of the discretized system as

$$
[K]\left\{d_{r}\right\}=\left\{r_{1}\right\}+\left\{r_{r_{2}}\right\},
$$

where

$$
\begin{aligned}
& {[K]=\left[K_{r r}\right]-\left[K_{r e}\right]\left[K_{e e}\right]^{-1}\left[K_{e r}\right]} \\
& \left\{r_{1}\right\}=\left\{r_{r}\right\}-\left[K_{r e}\right]\left[K_{e e}\right]^{-1}\left\{r_{e}\right\} .
\end{aligned}
$$

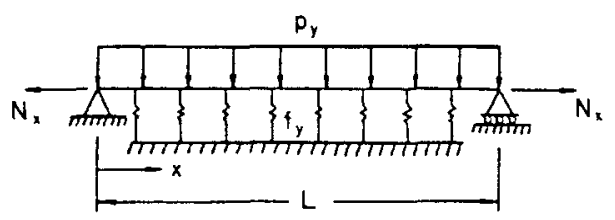

$L=300 \mathrm{in}$

$N_{n}=0$ or $4000 \mathrm{klb}^{+}$

$f_{y}=05 \mathrm{klb} / \mathrm{in}^{2}$

$p_{y}=0.39607 \mathrm{klb} / \mathrm{in}$.

$E^{y}=30000 \mathrm{klb} / \mathrm{in}^{2}$

$G=12000 \mathrm{klb} / \mathrm{in}^{2}$

$k_{z}=10 / 9$

$+k l b=100016$

Fig. 9. Simply supported beam for examples 1 and 2
If needed, the internal degrees of freedom $\left\{d_{e}\right\}$ can be calculated from the equation

$$
\left\{d_{e}\right\}=-\left[K_{e e}\right]^{-1}\left(\left[K_{e r}\right]\left\{d_{r}\right\}-\left\{r_{e}\right\}\right) .
$$

The discretized system of equations of the beam elements for both planes can be obtained by assembling the element equations [eqn (20)].

\section{NUMERICAL EXAMPLES AND} DISCUSSION

\section{Example I}

The accuracy of the proposed beam element on an elastic foundation is illustrated by solving the problem of a simply supported beam on a uniform, continuous elastic foundation under both a uniform load and a tensile axial force as in Fig. 9. The beam is of uniform circular cross section and is loaded only on its vertical plane $\left(p_{y} \neq 0, p_{z}=0\right)$; the only non zero elastic foundation constant in this case is $f_{y}$. All the numerical data for the specific example are shown in Fig. 9.

The boundary value problem which describes the displacement field of the example beam is

$$
\begin{gathered}
E I_{z}\left(1+\frac{k_{z} N_{x}}{A G}\right) \frac{\mathrm{d}^{4} v}{\mathrm{~d} x^{4}}-\left(N_{x}+\frac{E I_{z} k_{z}}{A G} f_{y}\right) \\
\times \frac{\mathrm{d}^{2} v}{\mathrm{~d} x^{2}}+f_{y} v=D_{।} \\
v(0)=v(L)=0 \\
M_{z}(0)=M_{z}(L)=0
\end{gathered}
$$

where

$$
\begin{aligned}
M_{z}(x)= & -E I_{z}\left(1+\frac{k_{z} N_{x}}{A G}\right) \frac{\mathrm{d}^{2} v}{\mathrm{~d} x^{2}} \\
& +\frac{E I_{z} k_{z}}{A G} f_{y} v-\frac{E I_{z} k_{z}}{A G} p_{y} .
\end{aligned}
$$

This boundary value problem was solved analytically in a straightforward manner as in [1]. The results for the data of Fig. 9 are tabulated in Table 1. The same problem was solved numerically using the beam element developed above using both 10 and 30 elements along the length of the beam. These results, also shown in Table 1, are in very good agreement with the analytical solution, especially those obtained with 30 elements. This provides an adequate indication that the element developed here does give a solution which converges to the exact solution as the number of elements increases.

If the displacement field must be calculated very accurately, a relatively large number of quadratic elements is required. For this reason, the more 
Table 1 .

\begin{tabular}{clll}
\hline & \multicolumn{3}{c}{ Displacement $v$ (in.) } \\
\cline { 2 - 4 }$x$ (in.) & $\begin{array}{c}\text { Analytic } \\
\text { solution }\end{array}$ & $\begin{array}{c}\text { Finite element } \\
\text { solution using } \\
\text { 10 elements }\end{array}$ & $\begin{array}{c}\text { Finite element } \\
\text { solution using } \\
\text { 30 elements }\end{array}$ \\
\hline 0.0 & 0.0 & 0.0 & 0.0 \\
30.0 & $0.0439479 \dagger$ & 0.0438395 & 0.0438992 \\
& $0.0391956 \ddagger$ & 0.0391459 & 0.0391949 \\
60.0 & 0.0830262 & 0.0828298 & 0.0829338 \\
& 0.0739946 & 0.0739096 & 0.0739934 \\
90.0 & 0.1135289 & 0.1132676 & 0.1134020 \\
& 0.1011101 & 0.1010017 & 0.1011085 \\
120.0 & 0.1328564 & 0.1325553 & 0.1327074 \\
& 0.1182679 & 0.1181462 & 0.1182661 \\
150.0 & 0.1394668 & 0.1391544 & 0.1393123 \\
& 0.1241337 & 0.1240078 & 0.1241319 \\
\hline
\end{tabular}

† Bending, clastic foundation, and shear cffects $\left(N_{r}=0\right)$.

\$ Bending, elastic foundation, shear and axial effects $\left(N_{x}=4000 \mathrm{klb}\right)$.

accurate quadratic element developed here is preferred over the simple linear element. High accuracy in calculating the displacement field is required in the rotor dynamics area, where a rotor displacement of even a few microinches $\left(10^{-6}\right.$ in.) changes the characteristics of the support bearings considerably.

\section{Example 2}

A simple supported beam on elastic foundation with a circular cross sectional area, as that of Fig. 9, is used to demonstrate the importance of coupling the shear, axial and elastic foundation effects in the bending of beams. Different beam length $L$ and diameter $D$, axial load $N_{x}$ and elastic foundation spring constant $f_{y}$ are used.

The shear effect depends on the shear correction factor $k$ and the product of the beam cross sectional area $A$ with the shear modulus $G$ [eqn (7)]. Since $G$ is constant for a selected material and $k$ does not vary considerably with the geometry of the beam cross section [19,22], the shear effect is governed mainly by the area $A$. For large $A$ (large diameter $D$ ) the shear effect is not important, while for small $D$ the shear effect is quite considerable (Table 2). Besides, the shear effect is more pronounced for beams with small $L / D$ ratios (see [16, 19, 23] and Table 2). Therefore, a combination of small $L / D$ ratios and small $D$ results in a large shear effect.

Table 2.

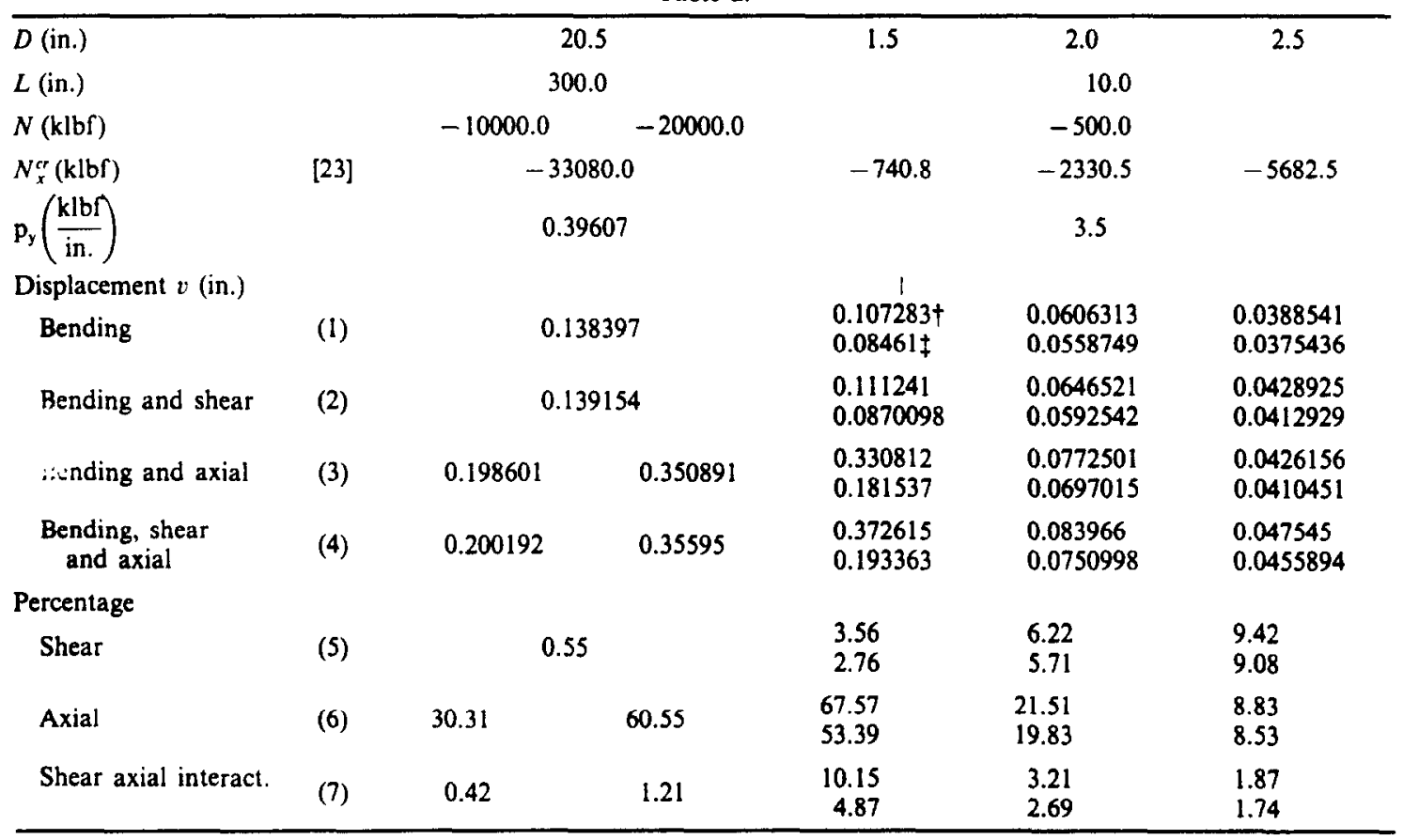

$(5)=\left|\frac{(2)-(1)}{(2)}\right| \times 100$.

$(6)=\left|\frac{(3)-(1)}{(3)}\right| \times 100$.

$(7)=\left|\frac{\{[(2)-(1)]+[(3)-(1)]-(1)\}-(4)}{(4)}\right| \times 100$.

$+f_{\mathrm{y}}=0.5 \mathrm{klbf} / \mathrm{in}^{2}$.

$\ddagger f_{y}=20.0 \mathrm{klbf} / \mathrm{in}^{2}$. 
It is well known that the deflection of a beam in bending due to the axial load $N_{x}$ is large when $N_{x}$ approaches the critical axial load $N_{x}^{c r}[23]$. In this case, the shear, axial interaction effect is practically important and it may be even greater than the pure shear effect (column 3 of Table 2). Generally, the shear, axial interaction effect is of significant magnitude when the shear effect, or the axial effect (or both) are practically considerable (Table 2 ).

The remarks stated above are also valid for different values of the elastic foundation spring constant $f_{y}$ (last three columns of Table 2). However, for large $f_{y}$ the beam is stiffer and, therefore, all the effects become less important (Table 2).

In conclusion, the shear effect for beams on elastic foundation is important for small $L / D$ ratios and/or small cross sectional areas, the axial effect is very important for axial loads of the same order of magnitude with the critical (buckling) load, and the shear, axial interaction is practically important when either the shear effect or the axial effect (or both) is important. However, the importance of the above effects decreases with increasing magnitude of the elastic foundation spring constants.

\section{Example 3}

The rotor of Fig. 10 was selected to demonstrate the importance of the proposed beam element on elastic foundation in the area of rotor dynamics. The problem of finding the response of a flexible rotor in its bearings is time dependent. However, the proposed element can be easily extended to include the dynamic effects of lateral and rotational inertias [13].

The beam element on elastic foundation is used to model only the part of the rotor which is within the journal bearings. The remaining rotor is modeled with beam elements which include bending, shear, axial and shear-axial interaction effects [22]. For these beam elements, the displacement field in each plane is approximated by a cubic polynomial using the displacement and rotation at both element ends as degrees of freedom. When the beam is not on elastic foundation, the vertical and horizontal planes are not coupled.

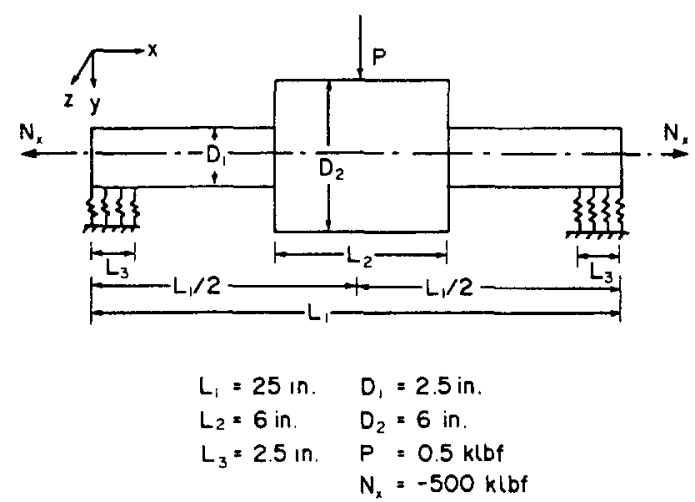

Fig. 10. Flexible rotor on elastic foundation for example 3.
The rotor of this example is supported by two hydrodynamic journal bearings. Both bearings have a length/diameter ratio of 1.0 and a radial clearance of $0.0006 \times D$. The rotor is turning at $1000 \mathrm{rev} /$ min. The bearings support, besides the rotor weight, two concentrated loads of $0.5 \mathrm{klbf}$ each applied at the rotor midspan in the vertical and horizontal planes. A compressible load of $500.0 \mathrm{klbf}$ is also applied on the rotor (Fig. 10).

Figures 6.9 and 6.10 of [24] are used to estimate the elastic foundation spring constants of the bearings' oil film corresponding to the above operating conditions. These estimated spring constants are as follows:

$$
\begin{array}{ll}
f_{y}=16.9 \mathrm{klbf} / \mathrm{in}^{2} & f_{y z}=404.5 \mathrm{klbf} / \mathrm{in}^{2} \\
f_{z}=37.1 \mathrm{klbf} / \mathrm{in}^{2} & f_{z y}=-404.5 \mathrm{klbf} / \mathrm{in}^{2} .
\end{array}
$$

The displacement, shear force and bending moment distributions of the rotor are shown in Figs 11, 12 and 13, respectively, for the vertical plane only. Similar distributions hold for the horizontal plane. In order to study the effect of the coupling elastic foundation

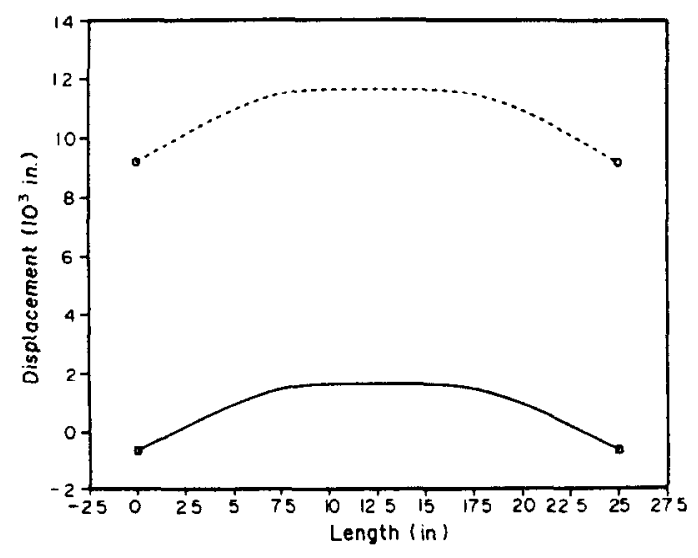

Fig. 11. Displacement distribution for the rotor of Fig. 10 in vertical plane.

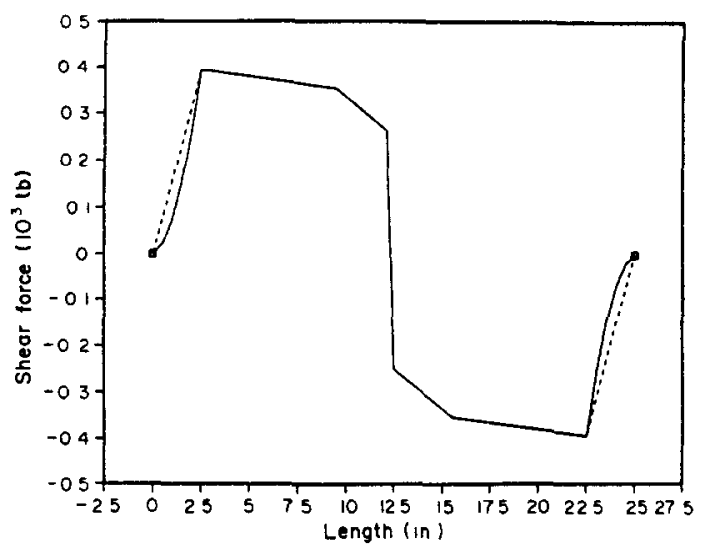

Fig. 12. Shear force distribution for the rotor of Fig. 10 in vertical plane. 


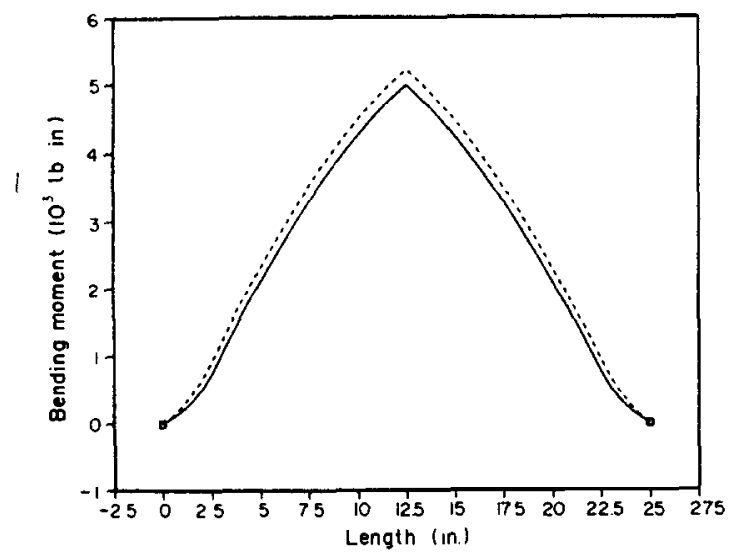

Fig. 13. Bending moment distribution for the rotor of Fig. 10 in vertical plane.

spring constants, each figure has two curves; one with $f_{y}, f_{z}, f_{y z}, f_{z y} \neq 0$ and one with $f_{y}, f_{z} \neq 0, f_{y z}=f_{z y}=0$. The effect of the coupling elastic foundation spring constants on the displacement field is very large, as shown in Fig. 11. When $f_{y z}=f_{z y}=0$, the elastic foundation deforms more in order to support the applied load. The shear force distribution is different, for the two cases, along the bearings' length (Fig. 12). However, the shear force is the same along the part of the rotor which is between the two bearings since the bearing reactions are the same for both cases. The difference in the shear force distribution along the bearings' length introduces also a difference in the bending moment distribution along the whole rotor length (Fig. 13). The difference for both the shear force and bending moment distributions is not as pronounced as it is for the displacement distribution because the bearings support the same load for both cases.

In conclusion, the effect of the coupling elastic foundation between the vertical and horizontal planes is very important when the rotor deformation is of primary concern (rotor dynamics area), and less important for the shear force and bending moment distributions, when a given load is applied on the rotor.

Acknowledgement-The authors are grateful to the reviewers for their constructive criticism which helped to improve the presentation of the paper.

This publication is a result of work sponsored by the Michigan Sea Grant College Program, project number R/T-17, under grant number NA80-AA-D-00072 from the Office of Sea Grant, National Oceanic and Atmospheric Administration (NOAA), U.S. Department of Commerce, and funds from the State of Michigan. The U.S. Government is authorized to produce and distribute reprints for governmental purposes notwithstanding any copyright notation appearing hereon.

\section{REFERENCES}

1. M. Eisenberger and D. Z. Yankelevsky, Exact stiffness matrix for beams on elastic foundation. Comput. Struct. 21, 1355-1359 (1985).

2. M. Eisenberger, D. Z. Yankelevsky and M. A. Adin, Moment distribution method for beams on elastic foundation. Civ. Engng Practicing Des. Engrs 4, 505-519 (1985).

3. M. A. Adin, D. Z. Yankelevsky and M. Eisenberger, Analysis of beams on bi-moduli elastic foundation. Comput. Meth. appl. Mech. Engng 49, 319-330 (1985).

4. B. Y. Ting and E. F. Mockry, Beams on elastic foundation finite element. J. Struct. Engng 110, 2324-2339 (1984).

5. F. Zhaohua and R. D. Cook, Beam elements on two-parameter elastic foundations. J. Engng Mech. 109, 1390-1402 (1983).

6. A. D. Kerr, Elastic and viscoelastic foundation models. J. appl. Mech. 31, 491-499 (1964).

7. M. Hetenyi, Beams on Elastic Foundations. University of Michigan Press, Ann Arbor, Ml (1946).

8. D. Q. Fletcher and L. R. Herrmann, Elastic foundation representation of continuum. J. Engng Mech. Div., ASCA 97, 95-107 (1971).

9. T. Y. Yang, A finite element analysis of plates on a two parameter foundation model. Comput. Struct. 2 , 593-614 (1972).

10. R. T. Severn, The solution of foundation mat problems by finite-element methods. Struct. Engr 44, 223-228 (1966).

11. Z. P. Mourelatos, M. G. Parsons and Z. Karni, Steadystate equilibrium of a flexible shaft in a hydrodynamic journal bearing. $J$. Tribology 109, 374-375 (1987).

12. Z. Karni, M. G. Parsons and Z. P. Mourelatos, Timevarying behavior of a statically indeterminate shafting system in a hydrodynamic journal bearing. $J$. Tribology 109, 115-123 (1987).

13. Z. Karni, Time varying lateral response of a marine shafting system within a stern bearing. Ph.D Dissertation, The University of Michigan, Ann Arbor, MI (1986).

14. O. C. Zienkiewicz, The Finite Element Method in Structural and Continuum Mechanics. McGraw-Hill, London (1967).

15. R. H. Gallagher, Finite Element Analysis, Fundamentals. Prentice-Hall, Englewood Cliffs, NJ (1975).

16. R. D. Cook, Concepts and Applications of Finite Element Analysis, 2nd edn. John Wiley, New York (1974).

17. R. T. Severn, Inclusion of shear deflection in the stiffness matrix for a beam element. J. Strain Anal. 5, 239-241 (1970).

18. R. E. Sandstrom, Informal notes for NA410-ship strength II. Department of Naval Architecture and Marine Engineer, The University of Michigan, Ann Arbor, MI.

19. J. T. Oden and F. A. Ripperger, Mechanics of Elastic Structures, 2nd edn. McGraw-Hill, New York (1981).

20. G. Strang and G. J. Fix, An Analysis of the Finite Element Method. Prentice-Hall, Englewood Cliffs, NJ (1973).

21. W. Kaplan, Advanced Mathematics for Engineers. Addison-Wesley, Reading, MA (1981).

22. Z. P. Mourelatos, Finite element analysis of elastohydrodynamic stern tube bearings. Ph.D. Dissertation, The University of Michigan, Ann Arbor, MI (1985).

23. S. P. Timosenko and J. M. Gere, Theory of Elastic Stability, 2nd edn. McGraw-Hill, New York (1961).

24. N. F. Rieger and J. F. Crofoot, Vibration of Rotating Machinery. Part I: Rotor-Bearing Dynamics. The Vibration Institute, Clarendon Hill, IL (1977). 